In Lateinamerika wird der überwiegende Anteil des Siedlungsabfalls ohne jegliche Vorbehandlung deponiert. Die Recyclingquote ist relativ gering, man ist aber bestrebt, diese Quote zu erhöhen. Insbesondere in den großen Städten hat der informelle Sektor den größten Anteil an der Recyclingquote. Vereinzelt gibt es kleinere Projekte zur Abtrennung und anschließenden Kompostierung oder Vergärung der organischen Fraktion im Siedlungsabfall. Eine Verbrennung von Siedlungsabfällen in Müllverbrennungsanlagen findet nicht statt.

Bei der Zersetzung der organischen Fraktion der Siedlungsabfälle in Deponien entstehen Deponiegase (im wesentlichen Kohlendioxid und Methan), die zum Treibhauseffekt beitragen. Zur Reduzierung der Freisetzungsmenge können die Deponiegase in Rohrleitungen gefasst und anschließend verbrannt werden. Diese Technologie wird im Rahmen von Projekten im Rahmen der Clean-Development-Mechanism-Maßnahmen finanziell gefördert und wurde daher in den letzten Jahren bereits auf einigen Deponien, so z. B. in Santiago de Chile, realisiert. Eine energetische Nutzung der gesammelten Deponiegase bietet zwar weitere ökologische Vorteile, wird aber bisher nicht umgesetzt, da sie sich i. a. finanziell nicht rentiert. Die einzelnen Vorträge können unter http://www.uninorte.edu.co/residuosredisa/ secciones.asp? $\mathrm{ID}=187$ eingesehen werden.

$\langle\langle\rangle$

\section{Im Fokus: Interdependenzen zwischen Technischem und Kulturellem}

\author{
Einführende Bemerkung zur KIT-Re- \\ levanz der im Folgenden vorgestellten \\ Tagungsthemen
}

\section{von Gerhard Banse, ITAS}

Die wechselseitigen Beziehungen zwischen Technik und Kultur werden in diversen wissenschaftlichen Disziplinen in zunehmendem Maße reflektiert. In der Technikphilosophie und -soziologie, in den Kulturwissenschaften oder in der Interkulturellen Kommunikationswissenschaft sind unterschiedliche Verständnisse von Technik und Kultur zugrunde gelegt, z. B. wird von „Technik und/als Kultur“, von der „Kulturalität des Technischen“, von der „Technizität des Kulturellen“ oder von „Kultürlichkeit der Technik“ gesprochen. Auch wird das Kulturelle im Vergleich etwa zum Sozialen als „ResidualKategorie" verstanden.

Hinter all diesen verschiedenen Ansätzen steht als gemeinsames erkenntnisleitendes Interesse, Zusammenhängen nachzuspüren, die etwa durch globalen Techniktransfer oder Kommunikationsprozesse mit den bisherigen wissenschaftlichen Ansätzen nicht oder nicht ausreichend in den Blick gerieten. Diesem Trend wollen sich das KIT und ITAS nicht verschließen. Im Gegenteil, es wird initiativreich agiert: Im KIT gibt es den Kompetenzbereich „Technik, Kultur und Gesellschaft“, u. a. mit den Kompetenzfeldern „Kulturerbe und sozialer Wandel“" sowie „Wechselwirkung von Wissenschaft, Technik und Gesellschaft". Dieses Kompetenzfeld hat eine Forschergruppe „Technik und Kultur“. Und der KITSchwerpunkt „Mensch und Technik“ beinhaltet auch den Topic „Kultur und Technik“. ITAS ist darin von Anfang an integriert, denn im Helmholtz-Programm ,Technology, Innovation \& Society. Topic 1: Science and Society: Challenges and Expectations“ ist die Untersuchung der ,relation between technologies and their impact on cultural patterns" als Aufgabe festgeschrieben. 
Die drei nachfolgenden Tagungsberichte verdeutlichen Aktivitäten in diesem Forschungsgebiet auf je unterschiedliche Weise: Heinrich Ganthaler informiert über die Jahrestagung 2009 eines internationalen Netzwerkes, das es sich zur Aufgabe gemacht hat, interdisziplinär Veränderungen kultureller Praxen (etwa Nutzungsmuster, Nutzungsmotivationen und Nutzungssituationen), die im Zusammenhang mit der Anwendung der so genannten Neuen Medien stehen, zu untersuchen. Der Beitrag von Lucia Belyová und Katrin Geske bezieht sich auf eine Tagung, die im Rahmen eines KIT-Startup-Budgets ermöglicht wurde und deren Gegenstand kulturelle Aspekte von Techniksicherheit und Technikkommunikation waren. Jessica Heesen schließlich berichtet über eine Aktivität am KIT, die sich mit der Thematik der kulturellen Überlieferung angesichts der Dynamik (nicht nur) des technischen Wandels befasst. Aus allen Berichten wird zweierlei deutlich: Erstens sind die dargestellten Aktivitäten Beiträge zur weiteren Strukturierung der Forschungslandschaft. Zweitens zeigen die Beiträge über die eigentliche Tagungsberichterstattung hinaus, dass die Interdependenzen zwischen Technischem und Kulturellem zukünftig Gegenstand von Forschungsanträgen sein könnten und neue Formen von Vernetzung und Kooperation verlangen.

\section{Neue Medien und ihr Einfluss auf die kulturelle Entwicklung der Gegenwart}

\section{Cottbus, 27.-29. September 2009}

\section{von Heinrich Ganthaler, Universität Salzburg}

Wie beeinflussen Neue Medien die kulturelle Entwicklung der Gesellschaft und welchen Einfluss haben sie auf die Identität einer Gesellschaft? Welche Möglichkeiten des Wissenstransfers eröffnen sie und welche Gefahren sind damit verbunden? Fragen dieser Art stehen im Zentrum der Forschungsaktivitäten des European Research Networks on Cultural Diversity and New Media (CULTMEDIA). Ziel der Tagung in Cottbus, an der Wissenschaftler aus sechs Ländern (Deutschland, Finnland, Österreich, Polen, Slowakische Republik und Tsche- chische Republik) teilnahmen, war es, die Ergebnisse der bisherigen Tätigkeit des Netzwerks zu reflektieren sowie neuere Entwicklungen im Medienbereich und deren kulturelle Auswirkungen zu thematisieren. Organisiert wurde die Tagung von Irene Krebs von der Brandenburgischen Technischen Universität Cottbus (BTU), eröffnet wurde sie vom Präsidenten der BTU, Walther Chr. Zimmerli.

\section{Rückblick auf die bisherige Tätigkeit des Netzwerks}

Wie der Organisator des Netzwerks, Gerhard Banse (ITAS, Karlsruhe), in seiner Einführung rückblickend hervorhob, gingen der Cottbuser Tagung insgesamt acht Veranstaltungen (Tagungen oder Workshops) in verschiedenen Ländern Europas voran. ${ }^{1}$ Thematisch konzentrierten sich die Beiträge dieser Veranstaltungen ebenso wie der Tagung in Cottbus auf vier Schwerpunkte bzw. Forschungsfelder: Privatheit und Öffentlichkeit (Erforschung der soziopolitischen Dimension des Einflusses neuer Medien), Identität und Gemeinschaft (Erforschung der sozialkulturellen Dimension des Medieneinflusses), Wissen und Wirtschaft (Erforschung der sozioökonomischen Dimension bzw. des Einflusses neuer Medien auf das Verhältnis von Wissen und Wirtschaft) sowie (Un-)Sicherheit und Vertrauen (Thematisierung von Sicherheitsfragen im Hinblick auf elektronische Signaturen, Diebstahl von Identitäten, unautorisierte Veränderungen von Medieninhalten etc.). ${ }^{2}$

\section{Beiträge zur Tagung in Cottbus}

Wie Reima Suomi (Turku School of Economics, Finnland) in seinem Einleitungsreferat hervorhob, hat das Internet die Welt zu Beginn des 21. Jahrhunderts ebenso stark verändert wie die Erfindung der Dampfmaschine das 19. Jahrhundert und die Entwicklung von Autos, Flugzeugen, Telefon, Radio und Fernsehen das 20. Jahrhundert. Die Entwicklung des Internets hat v. a. zu einem Informationsüberfluss beigetragen, den wir heute kaum mehr bewältigen können, und auf den Menschen in unterschiedlicher 